\title{
Anémie chez les enfants de moins de 5 ans reçus en consultation au service de pédiatrie de l'Hôpital de Zone d'Abomey-Calavi/So-Ava (Sud du Bénin)
}

\author{
Adégnika Amirath ADEBO ${ }^{1}$, Abdou Ganiou YESSOUFOU 1*, Justin Gbessohele BEHANZIN1, Akotayé \\ Antoine KABANOUDE ${ }^{1}$, Abèbi Karimath YESSOUFOU 2 \\ 1 Laboratoire de Biomembranes et de Signalisation Cellulaire, Département de Physiologie Animale, Faculté \\ des Sciences et Techniques, Université d'Abomey-Calavi, BP 526 Cotonou, République du Bénin. \\ $2 \quad$ Clinique de Pédiatrie et Néonatologie de Sènadé 06 BP 601 Cotonou, République du Bénin. \\ *Auteur correspondant, E-mail : yessoufouga@yahoo.fr / yessoufou601@gmail.com ; Tél : (00229) 97441045/ \\ (00229) 61788161
}

Original submitted in on $1^{\text {st }}$ February 2018. Published online at www.m.elewa.org on $31^{\text {st }}$ March 2018 https://dx.doi.org/10.4314/jab.v123i1.6

\section{RESUME}

Objectifs : Le présent travail vise à étudier la dénutrition et l'anémie chez les enfants de moins de 5 ans reçus en consultation et/ou hospitalisés au service de pédiatrie de l'Hôpital de Zone Abomey-Calavi / So-Ava dans le Département de l'Atlantique au Sud du Bénin

Méthodes et Résultats : L'étude est portée sur 301 enfants reçus en consultation et/ou hospitalisés et leurs mères sont soumises à un questionnaire élaboré à cet effet. Les indices anthropométriques ont été calculés à l'aide du logiciel WHO Anthro® (Version 3.2.2). Les données biologiques ont été collectées dans les dossiers des enfants. $25,25 \%$ des enfants étaient émaciés, $24,25 \%$ accusaient un retard de croissance et $31,89 \%$ présentaient une insuffisance pondérale. Par ailleurs, $56,48 \%$ des enfants souffraient de l'anémie. Parmi ceuxci, $30 \%$ présentaient une anémie sévère, $13,53 \%$ une anémie modérée et $56,47 \%$ une anémie légère. $58,83 \%$ des enfants ont une anémie normochrome et $72,94 \%$ ont une anémie microcytaire. La cause principale de l'anémie identifiée est la carence en fer due à un apport insuffisant en fer et/ou une perte de fer par l'organisme causée par les infections parasitaires (paludisme, les vers intestinaux) et bactériennes.

Conclusion et Applications des résultats : II ressort de cette étude, que la dénutrition (sous ses différentes formes) et l'anémie nutritionnelle demeurent un problème de santé publique au sein de la population infantile de la Commune d'Abomey-Calavi. II est alors nécessaire de renforcer les séances de sensibilisation et une éducation nutritionnelle de la population sur les bonnes habitudes alimentaires et hygiéniques. Une politique en faveur de la supplémentation en fer, du contrôle de paludisme et du déparasitage systématique des enfants permettrait de réduire l'incidence de l'anémie.

Mots clés : Bénin, état nutritionnel, anémie nutritionnelle, carence en fer, éducation nutritionnelle 


\section{ABSTRACT \\ Anemia in children under 5 received in consultation at the pediatric ward of Abomey-Calavi / So-Ava Zone Hospital (Southern Benin)}

Objectives: The present work aims at studying malnutrition and anemia in children under 5 years of age received in consultation and / or hospitalized at the pediatric ward of Abomey-Calavi / So-Ava Zone Hospital in the Department of the Atlantic in southern Benin.

Methods and Results : The study is carried out on 301 children received in consultation and / or hospitalized and their mothers are subjected to a questionnaire elaborated for this purpose. Anthropometric indices were calculated using the WHO Anthro® software. Biological data were collected from the children's files. $25.25 \%$ of the children were emaciated, $24.25 \%$ were stunted and $31.89 \%$ were underweight. In addition, $56.48 \%$ of children suffered from anemia. Of these, $30 \%$ had severe anemia, $13.53 \%$ had moderate anemia, and $56.47 \%$ had mild anemia. $58.83 \%$ of children have normochromic anemia and $72.94 \%$ have microcytic anemia. The main cause of the identified anemia is iron deficiency due to an insufficient dietary intake of iron and / or a loss of iron by the body caused by parasitic infections (malaria, intestinal worms) and bacterial infections.

Conclusion and Applications of the results : It emerges from this study that malnutrition (in its various forms) and nutritional anemia remain a public health problem among the child population of the Commune of AbomeyCalavi. It is then necessary to reinforce the sensitization sessions and a nutritional education of the population on the good habits of food and hygienic. A policy favoring iron supplementation, and malaria control and deworming reduction systematic reduction of children would reduce the incidence of anemia

Key words : Benin, nutritional status, nutritional anemia, iron deficiency, nutritional education

\section{INTRODUCTION}

L'anémie demeure un problème de santé publique aussi bien dans les pays industrialisés que dans les pays en développement. Elle se définit par une diminution de la concentration d'hémoglobine en dessous des valeurs limites en rapport avec l'âge, le sexe et l'état physiologique des individus (El Hioui et al., 2006). L'anémie est l'un des problèmes de santé publique les plus répandus dans le monde, surtout dans les pays en voie de développement. II s'agit d'une maladie aux causes multiples, à la fois nutritionnelles (carences en vitamines et en minéraux) et non nutritionnelles (infections), qui surviennent fréquemment en parallèle (EI Hioui et al., 2007). L'anémie nutritionnelle, due à une carence en nutriments (fer, vitamines et autres), est fréquente dans beaucoup de pays du monde. Les pays en voie de développement connaissent les prévalences les plus élevées ; on y relève les taux de l'ordre de $60 \%$ chez les femmes enceintes, $50 \%$ chez les enfants de moins de 4 ans et $48 \%$ chez les enfants d'âge scolaire (El Hioui, 2009). Au Bénin, selon l'Enquête Démographique de Santé au Bénin 2011-2012 (EDSB-VI, 2013), plus de la moitié des enfants de 6 à 59 mois (58\%) sont atteints d'anémie dont $26 \%$ sous forme légère, $29 \%$ sous forme modérée et $3 \%$ sont atteints d'anémie sévère. Avec cette prévalence élevée et selon le seuil définit par l'OMS qui est de 40\% (McLean et al., 2007), l'anémie demeure un problème de santé publique sévère au Bénin. La fréquence toujours élevée de l'anémie laisse suggérer un échec des programmes mis en œuvre pour son éradication tels que le programme de distribution de moustiquaire (Apouey et al., 2016) et celui de l' approche intégrée pour un contrôle efficace de I anémie (OMS/UNICEF, 2005). Une des raisons clés de l'échec de ces programmes est que beaucoup ont été conçus selon l'hypothèse que la seule cause d'anémie était la carence en fer (Badham, 2007). Alors une étude continuelle sur l'anémie serait nécessaire afin de discerner les véritables causes de l'anémie. C'est dans ce cadre que s'inscrit notre étude intitulée "Prévalence de l'anémie chez les enfants de moins de cinq ans reçus dans le service de pédiatrie du Centre Hospitalier Universitaire de zone Abomey-Calavi/SôAva ». 


\section{MATERIEL ET METHODES}

C'est une étude prospective, descriptive et analytique, qui s'est déroulée de Décembre 2015 à Mars 2016, sur un échantillon de 301 enfants de moins de 5ans reçus en consultation et/ou hospitalisés dans le Service de pédiatrie l'Hôpital de Zone Abomey-Calavi / So-Ava et leurs mères soumises à un questionnaire. Sont inclus dans cette étude, tous les enfants dont les parents sont consentants avec suspicion ou non d'anémie clinique, ayant bénéficié ou non d'une numération ou d'un dosage de l'hémoglobine et de l'hématocrite et hospitalisé dans le service de pédiatrie durant la période d'étude. Les variables étudiées ont porté sur l'âge, le sexe, les mesures anthropométriques, les aspects cliniques des enfants (type de malnutrition, complications associées), la pratique d'allaitement exclusif et l'alimentation de complément des enfants, et le niveau d'instruction et la profession des parents.

Mesures anthropométriques: Les mesures anthropométriques ont été recueillies selon les procédures de l'OMS (OMS, 2008). Nous avons utilisé un pèse-bébé de type $A D E$, un pèse-personne de type SECA (précision de $100 \mathrm{~g}$ ), une toise horizontale et une toise verticale (précision de $1 \mathrm{~mm}$ ). L'âge a été déterminé soit à partir des extraits d'acte de naissance, soit à partir des carnets de santé des enfants et des carnets de maternité de la mère ou soit à partir des informations recueillies des mères des enfants. Les indices anthropométriques telles que : le poids-pour-âge (P/A), la taille-pour-âge $(T / A)$, le poids-pour-taille $(P / T)$ ont été calculés à l'aide du logiciel WHO Anthro® version 3.2.2. La classification des enfants selon les indices anthropométriques est la suivante:

-P/A : cet indice nous a permis de déterminer le pourcentage d'enfants ayant un poids insuffisant (P/A audessous de -2ET) et un poids gravement insuffisant (P/A au-dessous de $-3 \mathrm{ET}$ ).

-T/A : cet indice nous a permis de déterminer le pourcentage d'enfants atteints de retard de croissance (T/A au-dessous de -2 ET) et de retard important de croissance (T/A au-dessous de $-3 \mathrm{ET}$ ).

-P/T : cet indice nous a permis de déterminer le pourcentage d'enfants émaciés (P/T au-dessous de -2 $E T)$, gravement émaciés ( $P / T$ au-dessous de $-3 \mathrm{SD}$ ), ayant un risque possible de surpoids (P/T au-dessus de

\section{RESULTATS}

Sur un total de 301 enfants de moins de 5 ans reçus en consultation et/ou hospitalisés, ceux de sexe féminin sont surreprésentés avec une sex-ratio de 0,90 et plus de 2/3 avaient moins de 24 mois. II ressort des résultats que
$+1 \mathrm{ET})$, ayant un surpoids (P/T au-dessus de $+2 \mathrm{ET})$ et obèses (P/T au-dessus de $+3 \mathrm{ET})$.

Les données biologiques (goutte épaisse et densité parasitaire): Pour poser le diagnostic biologique de l'anémie chez les enfants, nous avons utilisé la grille des valeurs normales de l'hémogramme proposée par Guiraud (2010) qui définit un taux d'hémoglobine en fonction de l'âge. Les examens pour l'orientation étiologique demandés systématiquement ont été les suivants :

La Numération des Formules Sanguines (NFS), la Goutte Épaisse /Densité Parasitaire (GE/DP), la CRP.

Les paramètres de la lignée rouge utilisés pour la classification des anémies et les recherches étiologiques sont les suivants :

- Concentration Corpusculaire Moyenne en Hémoglobine (C.C.M.H).

La valeur normale de la CCMH est comprise entre 32-36 g/dl (base du laboratoire du CHU-Z AC/SA)

C.C.M. H : Normale $\rightarrow$ Anémie normo chrome

C.C.M. H : bas $\rightarrow$ Anémie hypochrome

- Volume Globulaire Moyen (VGM)

La valeur normale du VGM est comprise entre 80-90 fl.( base du laboratoire du CHU-Z AC/SA)

V.G.M. Normal $\rightarrow$ Anémie normocytaire

V.G.M. Bas $\rightarrow$ Anémie microcytaire

V.G.M. Élevé $\rightarrow$ Anémie macrocytaire

- Taux d'hémoglobine $(\mathrm{Hb})$ défini selon l'OMS (2008)

$\mathrm{Hb}$ compris entre $9 \mathrm{~g} / \mathrm{l}$ et $11 \mathrm{~g} / \mathrm{l} \rightarrow$ Anémie légère

$\mathrm{Hb}$ compris entre $8 \mathrm{~g} / \mathrm{l}$ et $9 \mathrm{~g} / \mathrm{l} \rightarrow$ Anémie modérée

$\mathrm{Hb}$ inferieur à $8 \mathrm{~g} / \mathrm{l} \rightarrow$ Anémie sévère

Analyses statistiques des données: Les données recueillies sont saisies et analysées sur les logiciels suivants : Microsoft Office Excel 2010, SPSS version 16.0. Un test de Khi deux est réalisé pour l'analyse des données avec un seuil de significativité de $5 \%$

Considérations éthiques : L'enquête a pu être réalisée après l'autorisation des responsables du Centre Hospitalier Universitaire de zone Abomey-Calavi / Sô-Ava (CHU-Z AC/SA). Les personnes enquêtées ont donné oralement leur consentement libre et sont d'accord pour leur participation à l'étude.

$31,89 \%$ des enfants présentaient une insuffisance pondérale dont $13,95 \%$ de cas sévères, $25,25 \%$ des enfants étaient émaciés dont $08,97 \%$ de cas sévères et $24,25 \%$ des enfants accusaient d'un retard de croissance 
dont $12,29 \%$ de retard important de croissance. L'anémie touche $56,48 \%$ des enfants enquêtés et plus de $2 / 3$ $(68,83 \%)$ des enfants anémiés avaient moins de 24 mois. L'anémie sévère est plus remarquable chez les enfants de 36 à 59 mois. Les recherches étiologiques ont montré que $72,94 \%$ des enfants ont une anémie microcytaire et
$25,29 \%$ d'anémie normocytaire (figure 1). Par ailleurs, $58,83 \%$ des enfants ont une anémie normochrome et $35,29 \%$ une anémie hypochrome (figure 2). Les anémies hypochromes représentent environ le tiers des anémies $(35,29 \%)$ avec une forte fréquence des anémies hypochromes microcytaires $(85,00 \%)$.

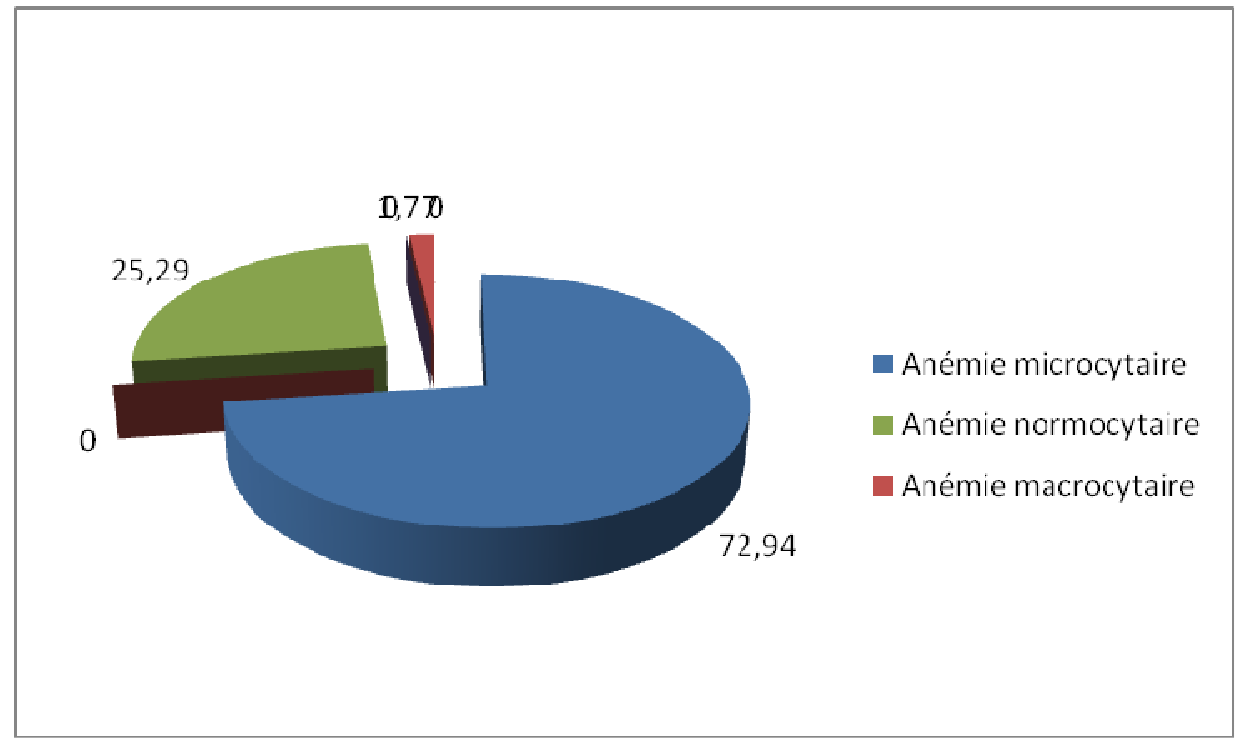

Figure 1 : Répartition des enfants selon le VGM

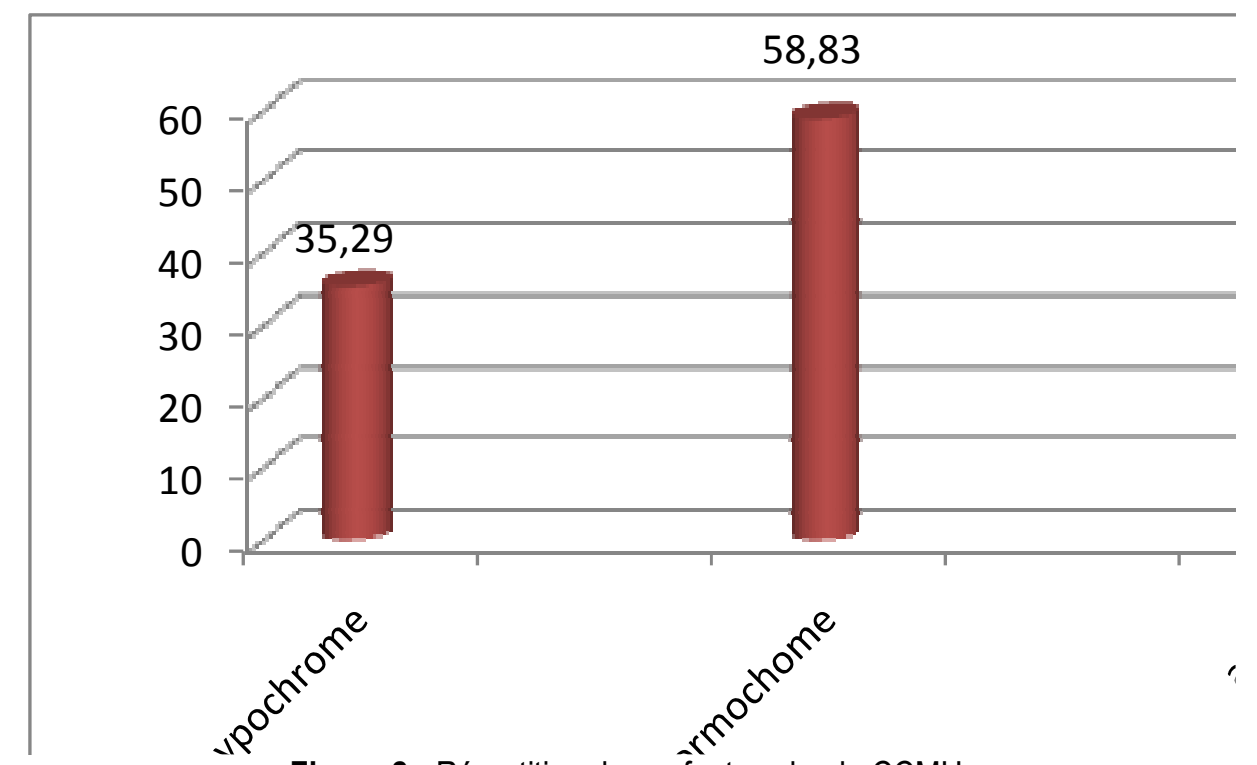

Figure 2 : Répartition des enfants selon le $\mathrm{CCMH}$ 


\section{DISCUSSION}

Dénutrition des enfants: Notre étude a révélé que $24,25 \%$ des enfants avaient un retard de croissance. Cette prévalence est inférieure à celle obtenue sur le plan national soit $37 \%$ et au niveau du département de l'Atlantique soit $36,3 \%$ (AGVSA, 2014). Cette différence peut s'expliquer par les caractéristiques de la population enquêtée (enfants reçus en pédiatrie dans une structure sanitaire) et la période où l'étude a été effectuée (période de post récolte pour la notre).

Anémie au sein de la population d'étude : II ressort de notre étude que $56,47 \%$ des enfants enquêtés souffraient d'une anémie. Cette prévalence est comparable à celle obtenue par l'enquête Démographique de Santé du Bénin (EDSB, 2013) (soit 58\%). Par ailleurs, la forme sévère de l'anémie est de $30 \%$ ce qui largement en dessus de celle de l'EDSB (2013). La forme légère est plus remarquée dans notre étude avec un taux de $56,47 \%$ des cas d'anémie. Ce résultat est proche de celui d'EL Hioui et al., (2009) avec $74,5 \%$. Le taux élevé de l'anémie légère pourrait s'expliquer du faite que notre étude a pris en compte tous les enfants de moins de cinq ans reçus en consultation durant la période d'étude

Causes de l'anémie: II ressort de nos résultats que $72,94 \%$ des enfants enquêtés présentaient une anémie microcytaire. Ce résultat est conforme à ceux obtenus par Yessoufou et al., (2015) et de ce qui est stipulé dans le profil nutritionnel du Bénin en 2011 (FAO, 2011). L'anémie microcytaire résulte d'une anomalie de synthèse de l'hémoglobine dans les érythroblastes, due surtout à l'indisponibilité de fer pour l'hémoglobinosynthèse ce qui nous permet d'attribuer la cause principale de l'anémie constatée à la carence en fer comme l'a souligné Sotto (2005). En effet, les besoins en fer sont élevés chez les jeunes enfants en particulier entre 6 et 18 mois, une fois que les réserves en fer de la naissance sont épuisées, le statut en fer des enfants dépend des aliments de complément ; malheureusement dans les pays en voie de développement, les aliments de complément traditionnels sont des piètres sources de fer biodisponibles. Par conséquent, les enfants de 6 à 18 mois sont fréquemment déficients en ce minéral comme l'a souligné Sena (2007). Presque le quart (25,29\%) des

\section{CONCLUSION}

En somme, l'anémie demeure un problème de santé publique sévère dans la commune d'Abomey-calavi. Une alimentation pauvre en fer biodisponible, des pertes du fer dues à des maladies infectieuses/parasitaires et la mauvaise absorption de fer, privent les enfants de fer dès leur petite enfance ce qui les expose à des anémies enfants souffraient d'une anémie normocytaire ce qui peut se traduit, soit par l'incapacité de la moelle osseuse à répondre aux besoins de production érythrocytaire (anémie normocytaire non régénérative) et/ou soit à une hémorragie récente ou une hyperhémolyse (anémie normocytaire régénérative). Les cas d'anémie macrocytaire observée au sein de la population d'étude seraient due à la carence en vitamine: carence en vitamine B12 ou carence en acide folique comme l'a souligné Scott (2007). Les anémies hypochromes normocytaires constatées ne sont probablement pas des anémies nutritionnelles. Par ailleurs, $61,78 \%$ des enfants enquêtés ont un CRP positif et $57,08 \%$ ont le GE/DP positif. Ainsi, plus de la moitié des enfants souffraient du paludisme dont les causes ne sont pas clairement élucidées dans notre étude car la majorité (2/3) des mères déclare utiliser les moustiquaires (imprégnés ou non). Néanmoins, n'est-il pas aisé de constater qu'un certain nombre de ménages défèquent leurs matières fécales à l'air libre et que plus du quart des ménages s'approvisionnent en eau de surface et autres. Les enfants sont ainsi exposés à des risques élevés d'infestation et d'infection causées par des agents pathogènes issus des sources d'eau non potables et des ordures qui sont à proximité des habitations comme l'ont souligné Brooker et al., (2007). Donc l'anémie serait aussi due à la perte de fer par l'organisme causée par les maladies parasitaires et infectieuses comme les ankylostomiases, les ascaridioses, les tuberculoses et l'infection par le VIH. La majorité des ménages dans lesquels vivent les enfants enquêtés ont une alimentation riche céréales et très pauvres en protéines et en fer héminique biodisponible (viande, poissons, œufs). Par ailleurs, la pauvreté de l'alimentation des ménages enquêtés en fruits et légumes nous permet de penser que l'anémie peut être due à une malabsorption de fer par l'organisme humain comme l'ont souligné plusieurs auteurs (Mohamed, 2013 ; Bencharif, 2011 ; Yessoufou et al., 2015). En effet, un régime riche en protéines d'origine animale, en fruits et légumes permet d'avoir un taux d'absorption de fer plus élevé de l'organisme humain comme l'ont souligné Badham et al., (2007).

microcytaires hypochromes constatées au sein de la population infantile de cette Commune. La mise en œurre d'une politique en faveur de la supplémentation en fer, de la diversification alimentaire des ménages, du contrôle du paludisme et du déparasitage systématique permettrait de réduire l'incidence des anémies. 


\section{REFERENCES}

AGVSA (Analyse Globale de la Vulnérabilité et de la Sécurité Alimentaire) 2014. Analyse globale de la vulnérabilité, de la sécurité alimentaire et de la nutrition. République du Bénin. p.142.

Apouey B., Picone G., Wilde J.,Coleman J.,Kibler R., 2016. Paludisme et anémie des enfants en Afrique subsahélienne : Effet de la distribution de moustiquaire. PSE Working; papers $\mathrm{n}^{\circ}$ 2016-02; 42p

El Hioui M, Ahami AOT, Aboussaleh Y, Lemrini JD, Loutfi H. 2006. Anémie en milieu hospitalier Marocain : Typologie et influences des facteurs sociodémographiques sur son incidence. Antropo., 12: 83-91.

El Hioui M, Ahami AOT, Aboussaleh Y, Rusinek S.,Dik K., Soualem A., 2007. L'anémie nutritionnelle chez les enfants scolarisés dans une zone rurale et côtière du Nord-Ouest marocain. Antropo., $15: 35-40$

El Hioui M, Aboussaleh Y, Ahami AOT, Farsi M. 2009. Contribution à l'Étude de la Prévalence de I'Anémie chez les Enfants Préscolaires de la Région de Kénitra. Antropo., 19: 1-5.

Erin McLean, Ines Egli, Mary Cogswell, Bruno de Benoist et Daniel Wojdyla 2007. Prévalence mondiale de l'anémie chez les enfants d'âge préscolaire, les femmes enceintes et les femmes en âge de procréer. Guide de l'anémie nutritionnelle. Edition Sight and Life Presse : Paris ; 11

Bencharif M., 2011 : Alimentation, état nutritionnel, apport calcique et calcémie d'une population de jeunes adultes. Mémoire de Master, Sciences Alimentaires, INATAA, Mentouri Constantine, $126 p$

Brooker, S., Jardim-Botelho, A., Quinnell, R. J., 2007 : Age-related changes in hookworm infection, anaemia and iron deficiency in an area of high Necator americanus hookworm transmission in south-eastern Brazil. Transactions of the Royal Society of Tropical Medicine \& Hygiene

EDSB-IV. 2013 : Enquête Démographique et de Santé (EDSB-IV) 2011-2012, Ministère du Développement de l'Analyse Économique et de la Prospective, INSAE, 573p

Guiraud G. 2010: Numération Formule Sanguine : Valeurs seuils. Variations non pathologiques, 7.

FAO. 2011. Profil Nutritionnel du Bénin : Division de la nutrition et de la protection des consommateurs, FAO.
Mohamed A., 2013: Prevalence and Risk Factors of Anemia among children 6-59 months old in Haiti. Article ID 502968, p 3.

OMS. 2008 : Cours de Formation sur l'Évaluation de la Croissance de l'Enfant. Genève : kit documentaire de 13 modules, OMS

OMS/UNICEF. 2005 : Déclaration conjointe de L'OMS et de l'UNICEF « focalisée sur l'anémie ; vers une approche intégrée pour un contrôle efficace de l'anémie ", Accessible sur http://www;who.int/topics/anaemia/who_unicef_a naemiastatement_fr.pdf

Senan L., 2007 : Métabolisme de Fer : Guide de l'anémie nutritionnelle ; Paris Edition Sight and Life Presse, 18-20.

Scott. J.M., 2007 : Les vitamines du groupe B : Guide de l'anémie nutritionnelle; Paris Edition Sight and Life Presse 24-26.

Sotto J. J., 2005 : Les anémies microcytaires par carence martiale- Corpus Medical- Faculté de Médecine de Grenoble en ligne http:// www-santé.uj fgrenoble. Fr/SANTE.

Yessoufou A. G., Béhanzin J., Ahokpè M., Djinti S. A., Bossou R., Sezan A., 2015 : La prévalence de l'anémie nutritionnelle chez les enfants malnutris de 6 à 59 mois hospitalisés dans le service de pédiatrie du Centre Hospitalier Départemental du Zou-Collines (CHD/Z-C) dans le plateau d'Abomey (Centre du Bénin). Int. Bio. Che. SCI 9(1) : 82-90 